\title{
Lipoxin A4 as a possible mediator of the beneficial actions of phosphodiesterase- 5 enzyme inhibitors
}

Undurti N. Das

UND Life Sciences, USA

Submitted: 3 April 2015

Accepted: 23 April 2015

Arch Med Sci 2017; 13, 1: 263-266

DOI: 10.5114 /aoms.2017.64723

Copyright $\odot 2016$ Termedia \& Banach

Phosphodiesterase-5 enzyme inhibitors (PDE-5-Is) such as sildenafil, vardenafil and tadalafil are used in the treatment of male erectile dysfunction (ED). Their beneficial action can be attributed to an increase in the intracellular cGMP level due to inhibition of PDE-5 and consequent increase of the levels of nitric oxide (NO), a potent vasodilator, anti-inflammatory agent and platelet anti-aggregator. In addition to their useful action in ED, PDE-5-Is have also been suggested to be of significant benefit in pulmonary hypertension, bronchial asthma, ischemic acute stroke, chronic obstructive pulmonary disease, pre-eclampsia and other diseases. In a recent study, El-Sayed and Amin [1] showed that administration of the PDE-5-Is sildenafil, vardenafil and tadalafil intravenously three times weekly for 4 weeks significantly increased PGF1 $\alpha$, calcium levels, prothrombin time (PT) and thrombin time (TT), and decreased TXB2 and fibrinogen levels compared to the control. Based on these results, the authors concluded that PDE-5-Is bring about their endothelial cyto-protective, thrombo-resistance anti-inflammatory and antinociception effects via activation of endothelial NOS (eNOS), increase of PGI2 synthesis and decrease of fibrinogen with significant increases in PT and TT. The results described by the authors El-Sayed and Amin are as expected, since PDE5 -Is are expected to enhance NO generation. The changes in the amount of generation of $\mathrm{NO}$ and differences shown by sildenafil, vardenafil and tadalafil with regard to their endothelial cyto-protective, thrombo-resistance anti-inflammatory and antinociception effects are not unexpected due to differences in their structure, half-life, pharmacodynamics and pharmacokinetics and their ability to enhance prostacyclin (PGI2) and/or decrease thromboxane A2 (TXA2) synthesis and action. In this context, I would like to suggest an alternative mechanism of action of PDE-5-Is that could explain both their beneficial effects and differences in their endothelial cyto-protective, thrombo-resistance anti-inflammatory and antinociception effects. I propose that the PDE-5-Is sildenafil, vardenafil and tadalafil either directly or by their ability to inhibit PDE are able to enhance formation of the anti-inflammatory, cytoprotective and antinociceptive molecule lipoxin A4 (LXA4).

Cis-linoleic acid (LA, 18:2 $\omega-6$ ) and $\alpha$-linolenic acid (ALA, 18:3 $\omega$-3) are called "essential fatty acids" (EFAs) since they are not formed in the body. Cis-linoleic acid is converted to $\gamma$-linolenic acid (GLA, 18:3, $\omega-6)$ by the enzyme $\Delta^{6}$ desaturase, and GLA, in turn, is elongated to form di-homo-GLA (DGLA, 20:3, $\omega-6$ ), the precursor of the 1 series of prostaglandins. DGLA can be converted to arachidonic acid (AA, 20:4, $\omega-6)$ by the enzyme $\Delta^{5}$ desaturase, the precursor of the 2 series of prostaglandins, thrombox-

\author{
Corresponding author: \\ Undurti N. Das MD, FAMS, \\ FRSC \\ UND Life Sciences \\ 2020 S $360^{\text {th }}$ St \\ \# K-202, Federal Way \\ WA 98003, USA \\ Fax: 2162315548 \\ E-mail: undurti@hotmail.com
}


anes and the 4 series leukotrienes (LTs). Arachidonic acid is also converted into non-enzymatic lipid mediators - 4-hydroxynonenal, isoprostanes, isoketals and isofurans - in tissues by free radicals in vivo and are useful as markers for oxidative stress.

Similar to $\omega-6$ LA, $\omega-3$ ALA is converted to eicosapentaenoic acid (EPA, 20:5, $\omega-3$ ) by $\Delta^{6}$ and $\Delta^{5}$ desaturases, the precursor of the 3 series of prostaglandins and the 5 series of LTs. Eicosapentaenoic acid can be elongated to form docosahexaenoic acid (DHA, 22:6, $\omega-3$ ). The AA, EPA and DHA also give rise to anti-inflammatory lipoxins: resolvins, protectins and maresins [2-11] (see Figure 1 for metabolism of EFAs and the formation of lipoxins and resolvins). Eicosanoids bind to $G$ protein-coupled receptors and mediate many steps of inflammation [7-10]. Non-steroidal anti-inflammatory drugs (NSAIDs) such as aspirin inhibit cyclo-oxygenase (COX) activity and thus bring about their anti-inflammatory action.

The general purpose of lipoxins and similar compounds generated from AA, EPA and DHA is not only to suppress the production of pro-inflammatory prostaglandins, thromboxanes, leu-

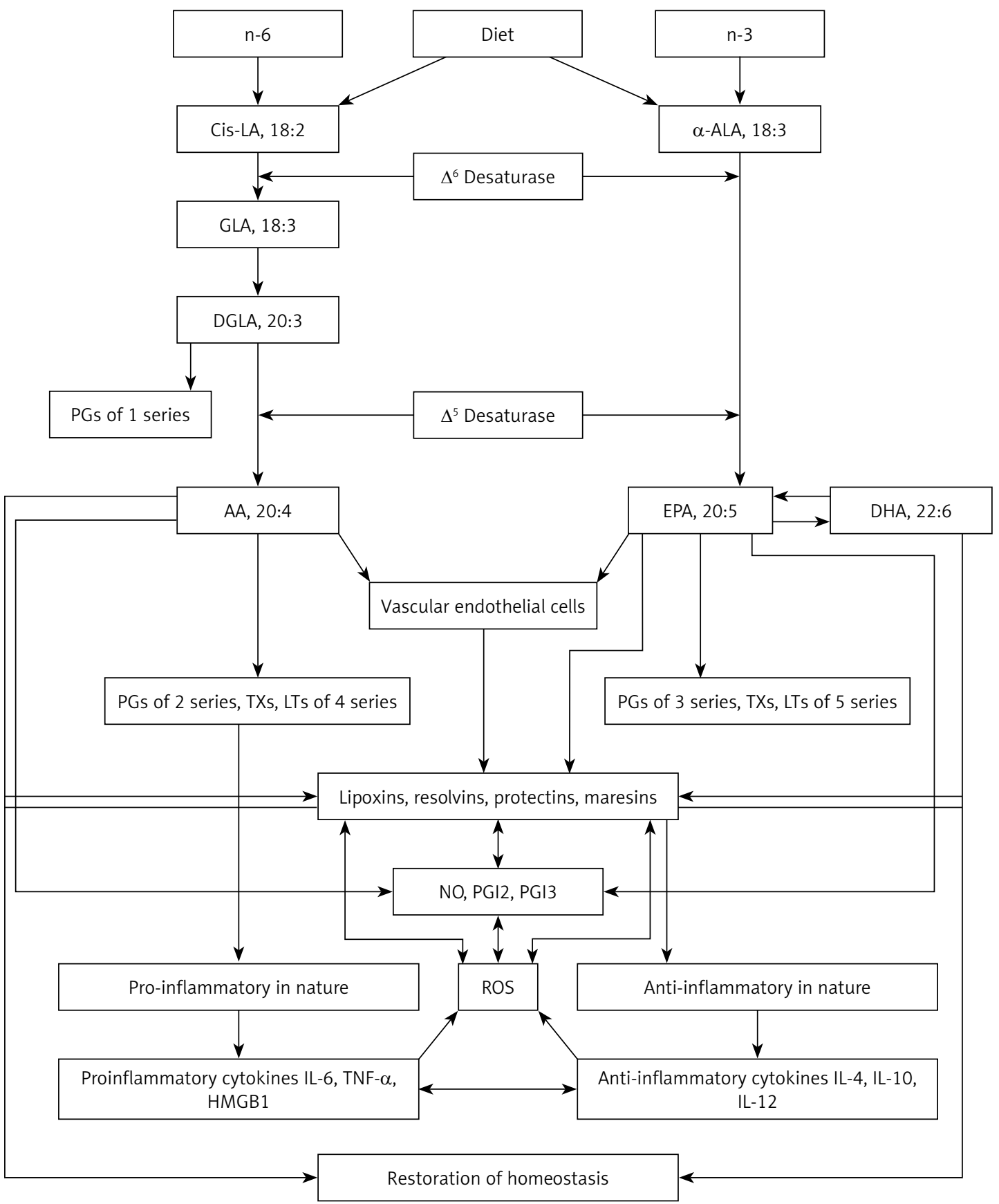

Figure 1. Schematic diagram showing metabolism of essential fatty acids, their role in inflammation and cytoprotection of endothelial cells 
kotrienes and isoprostanes but also to limit inflammation, enhance wound healing and resolve inflammation and thus restore cell function to normal. Hence, when the formation of lipoxins, resolvins, protectins and maresins is defective or subnormal, it could lead to persistent inflammation and tissue injury [6]. This implies that increased formation of lipoxins, resolvins, protectins and maresins protects various cells including endothelial cells against the cytotoxic action of interleukin-6 (IL-6), tumor necrosis factor- $\alpha$ (TNF- $\alpha$ ) and free radicals.

In this context, it is interesting to note that the beneficial effects of aspirin can be related to its inhibitory action on the enzyme cyclooxygenase (COX-2) and consequently a decrease in the production of pro-inflammatory prostaglandins (PGs), thromboxanes (TXs) and leukotrienes (LTS). It is noteworthy that aspirin does not interfere with the production of PGI2 but decreases the production of TXA2. In addition to inhibiting COX-2 activity, aspirin can also inhibit pro-inflammatory signaling pathways, gene expression and other factors distinct from eicosanoid biosynthesis that drive inflammation as well as enhance the synthesis of endogenous protective anti-inflammatory factors. Recent studies showed that aspirin induces the production of NO, which inhibits leukocyte/endothelium interaction during acute inflammation. Aspirin has the unique ability to acetylate the active site of inducible cyclooxygenase (COX-2), a property not shared with other NSAIDs, and generate the anti-inflammatory lipid mediators lipoxins (so-called aspirin-triggered epi-lipoxins), compounds that have profound roles in a range of host defense responses [12]. It was reported that a single anti-inflammatory dose of aspirin caused high levels of nitrite/nitrate to appear in the peripheral blood of the treated animals after a few hours. It was noted that a reduction in local inflammation correlated inversely with a dose-dependent and significant increase in plasma NO levels [13]. Further studies revealed that inhibiting aspirin-elicited NO nullified the anti-inflammatory effects of aspirin. Moreover, aspirin was not anti-inflammatory in either constitutive (eNOS) or inducible NO synthase (iNOS) knockout mice with IL-1 $\beta$-induced peritonitis. It was found that aspirin generated NO through its unique ability to trigger the synthesis of 15-epi-lipoxin A4. Aspirin and 15-epi-lipoxin A4 inhibited leukocyte trafficking in an NO-dependent manner. Both aspirin and 15-epi-lipoxin A4 markedly reduced effects on leukocyte-endothelial cell adherence in eNOS- and iNOS-deficient mice compared with wild type. This evidence led to the conclusion that aspirin acetylates COX 2 within the endothelium or circulating leukocytes to trigger 15 -epi-lipoxin
A4, which, in turn, elicits NO synthesis from both eNOS and iNOS. It is this aspirin-triggered NO that mediates the anti-inflammatory effects of aspirin in the microcirculation [13]. These results imply that one of the factors that regulate the production of NO is LXA4.

This is supported by the observation that the phosphodiesterase-III inhibitor cilostazol augments the infarct size (IS)-limiting effects of MK0626 (MK), a dipeptidyl-peptidase-4 (DPP4) inhibitor, by increasing intracellular CAMP in mice with type-2 diabetes. MK and cilostazol additive IS-limiting effects in diabetic mice were found to be associated with an increase in myocardial CAMP levels and protein kinase A (PKA) activity with downstream phosphorylation of Akt, eNOS, 5-lipoxygenase and CREB and downregulation of PTEN expression. It was shown that PKA phosphorylates 5-lipoxygenase at ser523, leading to increased production of 15-epi-lipoxin A4 [14-16].

Based on these results [12-16], I suggest that the beneficial actions of PDE-5-IS shown by ElSayed and Amin [1] are, at least in part, due to increased formation of LXA4 and, possibly, resolvins, protectins and maresins. This proposal could be verified by measuring plasma and tissue levels of LXA4 in those experimental animals and humans treated with sildenafil, vardenafil and tadalafil. This also implies that deficiency of AA, EPA and DHA (as would occur in EFA-deficient states) could interfere with the beneficial actions of sildenafil, vardenafil and tadalafil due to reduced formation of LXA4, resolvins, protectins and maresins. In addition, it is known that LXA4 has potent anti-nociceptive action similar to PDE-5-Is [17-19]. Based on the preceding discussion, it is concluded that further research is needed to confirm the role of LXA4, resolvins, protectins and maresins as one of the mechanisms of action of PDE-5-Is under different (patho)physiological conditions.

\section{Conflict of interest}

The author declares no conflict of interest.

\section{References}

1. El-Sayed MK, Amin HA. Mechanism of endothelial cyto-protective and thrombo-resistance effects of sildenafil, vardenafil and tadalafil in male rabbit. Arch Med Sci 2015; 11: 190-8.

2. Serhan CN. Lipoxins and aspirin-triggered 15-epi-lipoxins are the first lipid mediators of endogenous antiinflammation and resolution. Prostaglandins Leukot Essent Fatty Acids 2005; 73: 141-62.

3. Das UN. Essential fatty acids: bochemistry, physiology, and pathology. Biotechnol J 2006; 1: 420-39.

4. Serhan CN, Hong S, Gronert K, et al. Resolvins: a family of bioactive products of omega- 3 fatty acid transformation circuits initiated by aspirin treatment that counter proinflammatory signals. J Exp Med 2002; 196: 1025-37. 
5. Das UN. Essential fatty acids - a review. Curr Pharm Biotechnol 2006; 7: 467-82.

6. Das UN. Current and emerging strategies for the treatment and management of systemic lupus erythematosus based on molecular signatures of acute and chronic inflammation. J Inflammation Res 2010; 3: 143-70.

7. Lattin JE, Schroder K, Su Al, et al. Expression analysis of $\mathrm{G}$ protein-coupled receptors in mouse macrophages. Immunome Res 2008; 4: 5.

8. Milligan G, Stoddart LA, Brown AJ. G protein-coupled receptors for free fatty acids. Cell Signal 2006; 18: 1360-5.

9. Oh DY, Talukdar S, Bae EJ, et al. GPR120 is an omega-3 fatty acid receptor mediating potent anti-inflammatory and insulin-sensitizing effects. Cell 2010; 142: 687-98.

10. Bhagat U, Das UN. Potential role of dietary lipids in the prophylaxis of some clinical conditions. Arch Med Sci 2015; 11: 807-18.

11. Shen J, Bi YL, Das, UN. Potential role of polyunsaturated fatty acids in diabetic retinopathy. Arch Med Sci 2014; 10: 1167-74.

12. Claria J, Serhan CN. Aspirin triggers previously undescribed bioactive eicosanoids by human endothelial cell leukocyte interactions. Proc Natl Acad Sci USA 1995; 92: 9475-9.

13. Gilroy DW. New insights into the anti-inflammatory actions of aspirin induction of nitric oxide through the generation of epi-lipoxins. Mem Inst Oswaldo Cruz 2005; 100 (Suppl. I): 49-54.

14. Birnbaum Y, Castillo AC, Ling S, Bajaj M, Perez-Polo JR, Ye Y. Amplification of the myocardial infarct size limiting effects of exenatide with cilostazol, a phosphodiesterase III inhibitor. J Am Col Cardiol 2012; 59: A110.

15. Ye $Y$, Lin $Y$, Perez-Polo JR, et al. Phosphorylation of 5 -lipoxygenase at ser523 by protein kinase A determines whether pioglitazone and atorvastatin induce proinflammatory leukotriene B4 or anti-inflammatory 15 -epi-lipoxin a4 production. J Immunol 2008; 181: 3515-23.

16. Birnbaum Y, Castillo AC, Qian J, et al. Phosphodiesterase III inhibition increases CAMP levels and augments the infarct size limiting effect of a DPP-4 inhibitor in mice with type-2 diabetes mellitus. Cardiovasc Drugs Ther 2012; 26: 445-56.

17. Wang ZF, Li Q, Liu SB, et al. Aspirin-triggered lipoxin A4 attenuates mechanical allodynia in association with inhibiting spinal JAK2/STAT3 signaling in neuropathic pain in rats. Neuroscience 2014; 273: 65-78.

18. Jin H, Li YH, Xu JS, Guo GQ, Chen DL, Bo Y. Lipoxin A4 analog attenuates morphine antinociceptive tolerance, withdrawal-induced hyperalgesia, and glial reaction and cytokine expression in the spinal cord of rat. Neuroscience 2012; 208: 1-10.

19. Sun T, Yu E, Yu L, Luo J, Li H, Fu Z. LipoxinA(4) induced antinociception and decreased expression of NF-kappaB and pro-inflammatory cytokines after chronic dorsal root ganglia compression in rats. Eur J Pain 2012; 16 : 18-27. 